TITLE:

\title{
Usefulness of oils for cleaning the host matrix and for cryoprotection of lipidic cubic phase crystals
}

$\operatorname{AUTHOR}(S)$ :

Niwa, Satomi; Takeda, Kazuki

\section{CITATION:}

Niwa, Satomi ... [et al]. Usefulness of oils for cleaning the host matrix and for

cryoprotection of lipidic cubic phase crystals. Journal of Applied Crystallography 2019, 52: 864-868

ISSUE DATE:

2019

URL:

http://hdl.handle.net/2433/243225

RIGHT:

(C) International Union of Crystallography.; 許諾条件に基づいて掲載し ています。 


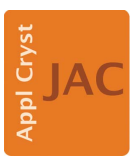

JOURNAL OF APPLIED CRYSTALLOGRAPHY

ISSN 1600-5767

Received 18 February 2019

Accepted 28 May 2019

Edited by F. Meilleur, Oak Ridge National Laboratory, USA, and North Carolina State University, USA

Keywords: lipidic cubic phase; cryocrystallography; membrane protein crystallography.

Supporting information: this article has supporting information at journals.iucr.org/j

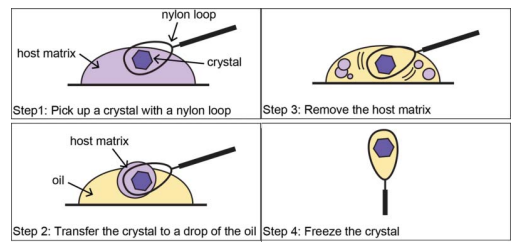

C 2019 International Union of Crystallography

\section{Usefulness of oils for cleaning the host matrix and for cryoprotection of lipidic cubic phase crystals}

\author{
Satomi Niwa and Kazuki Takeda* \\ Department of Chemistry, Graduate School of Science, Kyoto University, Sakyo-ku, Kyoto, Kyoto 606-8502, Japan. \\ ${ }^{*}$ Correspondence e-mail: ktakeda@kuchem.kyoto-u.ac.jp
}

The lipidic cubic phase method is an effective approach for membrane protein crystallography. The in meso grown crystals are usually cryocooled directly without removing the host matrix from the harvested crystal surface. However, the host matrix often causes the appearance of scattering rings and an increase in background scattering during the data collection. Moreover, the frozen host matrix sometimes becomes opaque and it can hinder conventional crystal centering. In this study, several oils were examined for their ability to clean the host matrix and to provide cryoprotection for crystals grown in the lipidic cubic phase. Several of the tested oils appeared to be useful in terms of their effect on crystal stability and background scattering. This method should be of value for the collection of highly accurate data sets.

\section{Introduction}

Membrane proteins play essential roles in various biological processes and have therefore attracted interest as potential drug targets. The lipidic cubic phase (LCP) or in meso method is an approach for crystallizing membrane proteins (Landau \& Rosenbusch, 1996; Pebay-Peyroula et al., 1997; Cherezov et al., 2007; Caffrey, 2015). This method provides type I crystals that are typically small but well diffracting (Michel, 1983). The crystals are grown in a host matrix, which is a material composed of water and lipids such as monoolein.

The in meso crystals are usually cryocooled directly after the harvest, because the host matrix acts as a cryoprotectant. Under some conditions, however, scattering rings are observed from the host matrix and ice. Moreover, the frozen host matrix sometimes becomes opaque in nylon loops. Sometimes the crystals obtained by the LCP method are very small and thus can be difficult to center using optical microscopy during a diffraction experiment. To overcome this situation, various methods have been proposed. The most popular method is using a micro-focused X-ray beam, such as the rastering for crystal mapping and the X-ray mesh scanning methods (Hilgart et al., 2011; Melnikov et al., 2018; Hirata et al., 2019). However, the availability of beamlines at which these methods using a micro-focused beam can be carried out is limited, and optical microscopy is still used during the crystal centering step at many beamlines. The crystal centering problem can thus impede the experiment and can also contribute to decreased diffraction intensity and increased background scattering. In some cases, the host matrix is removed from the crystals prior to data collection (Nollert \& Landau, 1998; Luecke et al., 1999). 
Table 1

Summary of oils used in this study.

\begin{tabular}{|c|c|c|c|}
\hline $\begin{array}{l}\text { Compound } \\
\text { name }\end{array}$ & Supplier & $\begin{array}{l}\text { Density } \\
\left(\mathrm{g} \mathrm{ml}^{-1}\right)\end{array}$ & $\begin{array}{l}\text { Viscosity } \\
\text { (cP) }\end{array}$ \\
\hline Squalane & Sigma (St Louis, MO, USA) & 0.81 & 27 \\
\hline Squalene & Sigma (St Louis, MO, USA) & 0.86 & 12 \\
\hline Phytantriol & Avanti Polar Lipids (Alabaster, AL, USA) & 0.93 & $-\dagger$ \\
\hline Paratone-N & $\begin{array}{l}\text { Hampton Research (Aliso Viejo, CA, } \\
\text { USA) }\end{array}$ & 0.87 & 550 \\
\hline Liquid paraffin & Nacalai Tesque (Kyoto, Japan) & 0.87 & 30 \\
\hline $\begin{array}{l}\text { Low-viscosity } \\
\text { liquid paraffin }\end{array}$ & Nacalai Tesque (Kyoto, Japan) & 0.82 & 3.4 \\
\hline
\end{tabular}

$\dagger$ Though the viscosity of phytantriol is unknown, it has high viscosity comparable to that of Paratone-N.

In data collection from soluble protein crystals, immiscible oils are sometimes used for cryoprotection (Riboldi-Tunnicliffe \& Hilgenfeld, 1999). These oils are suitable for crystals obtained under a wide range of crystallization conditions. In addition, the application of oils has been proposed for removing the host matrix from crystals obtained by the LCP method (Caffrey \& Cherezov, 2009). However, there has been no report of a systematic study of the use of oils for removal of the host matrix for cryoprotection, and the usefulness of oils in these contexts is unknown. In this study, we examined the effectiveness of several oils in the cryoprotection of crystals obtained by the LCP method in terms of the crystal quality and the facilitation of the experiment.

\section{Materials and methods}

\subsection{Materials}

The following six oils were chosen for these experiments: squalane, squalene, phytantriol, Paratone-N, liquid paraffin and low-viscosity liquid paraffin. The density and viscosity of each of the oils as given in their respective catalogs are listed in Table 1. Loops made with $20 \mu \mathrm{m}$-diameter nylon fibers were purchased from Hampton Research (Aliso Viejo, CA, USA).

\subsection{Background scattering}

For each of the oils, a small amount of oil was mounted on a nylon loop. The oil was then flash-cooled in a flowing nitrogen stream at a set point of $100 \mathrm{~K}$. X-ray diffraction images of each oil were collected at $100 \mathrm{~K}$.

\subsection{Crystal stability}

The effects on crystal stability were estimated using bacteriorhodopsin (bR) crystals which belong to space group $P 6_{3}$. This type of bR crystal has typical type I packing in which $2 \mathrm{D}$ layers of membrane proteins stack into a $3 \mathrm{D}$ crystal. bR was purified and crystalized as described previously (Hasegawa et al., 2018). Briefly, the purple membrane was purified from Halobacterium salinarum strain R1 (JMC9409) by a standard procedure (Oesterhelt \& Stoeckenius, 1971). bR was solubilized at $1.2 \%(w / v) \quad \beta$-octyl glucoside (Anatrace, Maumee, OH, USA) from the purple membrane. Crystals were obtained by the LCP method using monoolein as host matrix containing $1.0 \%(w / w)$ squalane (Sigma-Aldrich, $\mathrm{St}$ Louis, MO, USA) and 5\%(w/w) trehalose C16 (Dojindo, Kumamoto, Japan) as additives. Crystals were grown for more than three months in a precipitant solution containing 2-2.5 M $\mathrm{Na} / \mathrm{K}$ phosphate ( $\mathrm{pH}$ 5.6). Crystals with a largest dimension of 50-100 $\mu \mathrm{m}$ and a thickness of 10-20 $\mu \mathrm{m}$ were selected for the experiments, because this is a typical size for crystals obtained by the LCP method. The crystals and surrounding lipids were picked up with a nylon loop and transferred to a $1 \mu$ drop of the respective oil. Crystal cleaning was performed with the nylon loop by moving the crystal gently back and forth in the oil drop. The crystals were kept within the oil drops for $60 \mathrm{~min}$. Photographs of the crystals were taken at 1,5 and 60 min after transferring the crystal to the drop of oil.

\subsection{Data collection}

Initial diffraction experiments with all oils were performed using an in-house X-ray source with bR crystals cooled at $100 \mathrm{~K}$. Crystals of $\sim 80 \mu \mathrm{m}$ were transferred and cleaned in each oil. The crystals in the oil were immediately flash-cooled in a nitrogen gas stream at $100 \mathrm{~K}$. The steps of this method are shown in Fig. 1. Two diffraction images at two different angles $90^{\circ}$ apart were collected with an oscillation angle of $1^{\circ}$ and an exposure time of $5 \mathrm{~min}$. Further diffraction experiments with the selected oils were performed at BL41XU of SPring- 8 (Hyogo, Japan). Crystals of $50-150 \mu \mathrm{m}$ with the host matrix

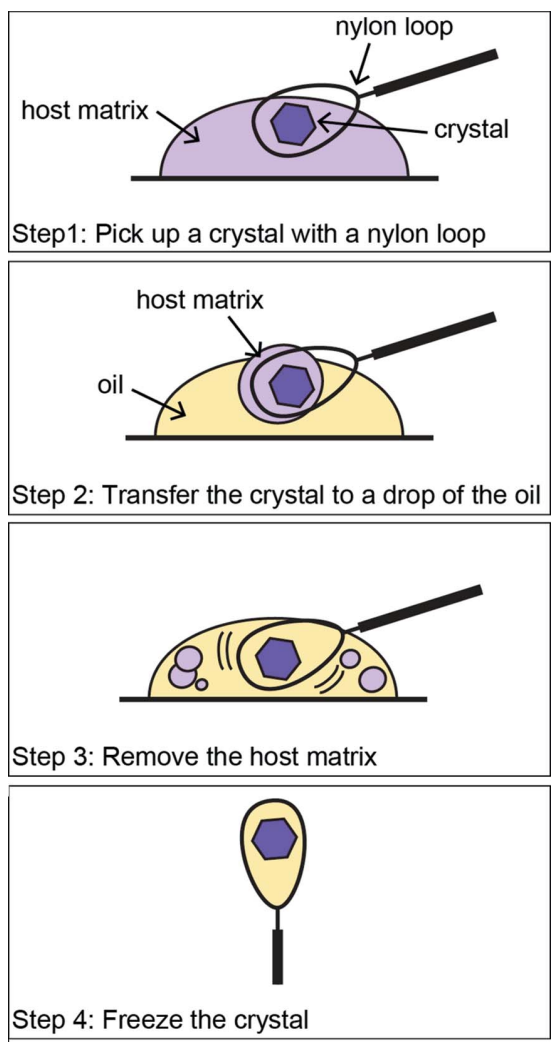

Figure 1

Steps of the crystal cleaning method. 
and treated with the respective oils were flash-cooled in a nitrogen gas stream at $100 \mathrm{~K}$. Data collections were performed with an oscillation angle of $0.2^{\circ}$ and an exposure time of $0.2 \mathrm{~s}$ for one frame, and a total of 500 frames were measured from each crystal with the selected oils. The wavelength of X-rays and the beam size were set to $0.80 \AA$ and $35 \times 22 \mu \mathrm{m}$, respectively. The photon flux was set to $2 \times 10^{10}$ photons s $^{-1}$ by using aluminium attenuators. Diffraction spots were recorded with an Eiger X 16M detector (Dectris). The distance between the sample and detector was set to $135 \mathrm{~mm}$. Crystals were cooled at $15 \mathrm{~K}$ with a helium gas stream during the data collection. The data sets were processed using the $X D S$ program (Kabsch, 2010). The appropriateness of data processing was evaluated by the value of $(I / \sigma)^{\text {asymptotic }}$, which is given as ISa in the $X D S$ program (Diederichs, 2010). Data sets at $(I / \sigma)^{\text {asymptotic }}<10$ were excluded from further analysis. The resolution limits were defined from $\mathrm{CC}_{1 / 2} \simeq 50 \%$. The Wilson $B$ factor, which reflects the diffraction quality of the crystals, was calculated using the ctruncate program (Dauter, 2006) in the CCP4 program suite (Collaborative Computational Project, Number 4, 1994).

\section{Results}

\subsection{Background scattering from oils}

Diffraction data were collected from a small amount of each oil as well as from the host matrix across the nylon loop. In the case of phytantriol and Paratone- $\mathrm{N}$, it is difficult to minimize the amount of liquid in the loop because of their high viscosity. A broad diffuse scattering ring was found in the range of 5$4.2 \AA$ in the diffraction pattern of each oil (Supplementary Fig. S1). In the diffraction image of phytantriol, this broad ring was especially strong, and an additional broad ring was observed in the low-resolution region of $\sim 20 \AA$. In the diffraction image of low-viscosity liquid paraffin, additional sharp rings were observed at around 4.1 and $3.6 \AA$. From these facts, we concluded that background scattering was negligible in squalane, squalene, Paratone-N and liquid paraffin but nonnegligible in phytantriol and low-viscosity liquid paraffin.

\subsection{Effect on crystal stability}

All oils were able to remove the host matrix from the crystal surface. The required time for cleaning was 1-5 min. Lowviscosity liquid paraffin dissolved and removed the host matrix most rapidly. Owing to their high viscosity, phytantriol and Paratone-N stripped the host matrix away from the crystals rather than dissolving it. Cleaning was most easily achieved with phytantriol and Paratone-N, and the second best were squalene and low-viscosity liquid paraffin.

After cleaning, these crystals were kept in their respective oil drops (Supplementary Fig. S2). At $5 \mathrm{~min}$, the edges of the crystals in squalene and low-viscosity liquid paraffin were dissolved. In the drop of phytantriol, the oil surrounding the crystals became clouded [Supplementary Fig. S2(c)]. No significant changes were observed in the other oils. After $60 \mathrm{~min}$, the crystals survived and retained their shape with the phytantriol and Paratone-N. However, the crystals cracked in the other oils. Squalene and low-viscosity liquid paraffin especially affected the crystals: the crystals disintegrated completely in these oils [Supplementary Figs. S2(b) and $\mathrm{S} 2(f)]$. In the drop of low-viscosity liquid paraffin, the water droplets merged to large droplets.

\subsection{Diffraction experiment}

The time required for cleaning and freezing one crystal was $\sim 3$ min. When phytantriol and Paratone-N were used, it proved very difficult to pick up the crystal and a small amount of oil in the loop owing to their high viscosity. Diffraction spots were observed for all crystals with oils as well as for the crystal in the host matrix which was directly picked up from the LCP (Supplementary Fig. S3). Ice rings were not found in any of the diffraction images. For squalane, phytantriol, Paratone-N and liquid paraffin, the resolution and mosaicity were comparable to those in the untreated crystal. On the other hand, the resolution was decreased with squalene and low-viscosity liquid paraffin. Moreover, the mosaicity was severely increased with low-viscosity liquid paraffin [Supplementary Fig. S3 $(g)$ ]. From these results, we concluded that crystals in squalene and low-viscosity liquid paraffin deteriorated quickly.

\subsection{Data collection at the synchrotron facility}

On the basis of these results, we selected squalane, Paratone-N and liquid paraffin for further assessment. As mentioned in Section 3.3, it was very difficult to mount crystals properly with Paratone-N. Moreover, the treatment with Paratone-N became more difficult when the crystals were smaller. In the case of squalane and liquid paraffin, even small crystals were picked up easily from the oil drops, and it was easy to align the orientation of the plane of the crystals to the nylon loops. The crystals treated with the respective oils in the nylon loops are shown in Fig. 2. In the case of no treatment, the crystals were difficult to observe with a microscope from the perpendicular direction of the nylon loop plane owing to the thick and opaque host matrix. For squalane, the crystal position was clearly visualized from an in-plane direction and the perpendicular direction of the nylon loop plane, which

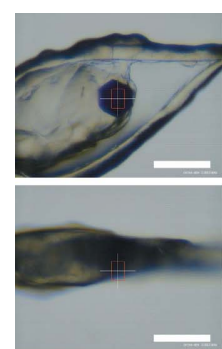

(a)

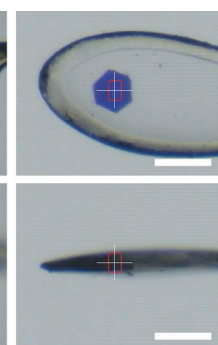

(b)

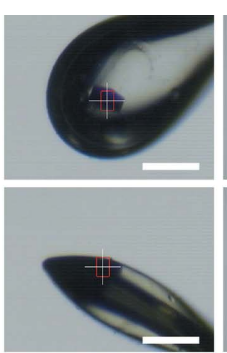

(c)

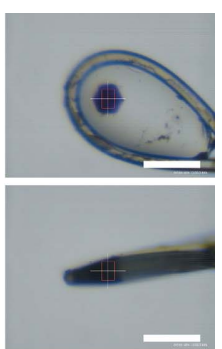

(d)
Figure 2

The crystals used for data collection with $(a)$ the host matrix, $(b)$ squalane, $(c)$ Paratone-N and $(d)$ liquid paraffin. The scale bars indicate $0.1 \mathrm{~mm}$. 
helped in the crystal centering. For Paratone-N, the crystal position was clearly visualized from an in-plane direction of the nylon loop plane. However, the crystal position was not clear from the perpendicular direction of the nylon loop plane owing to the large volume of Paratone-N [Fig. 2(c)]. Thus, the

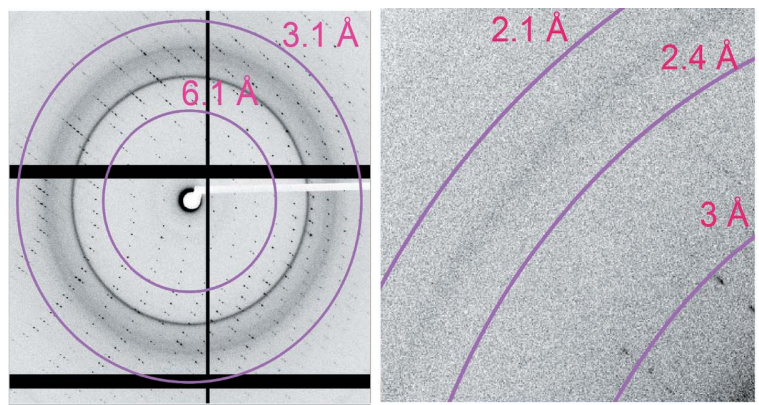

(a)

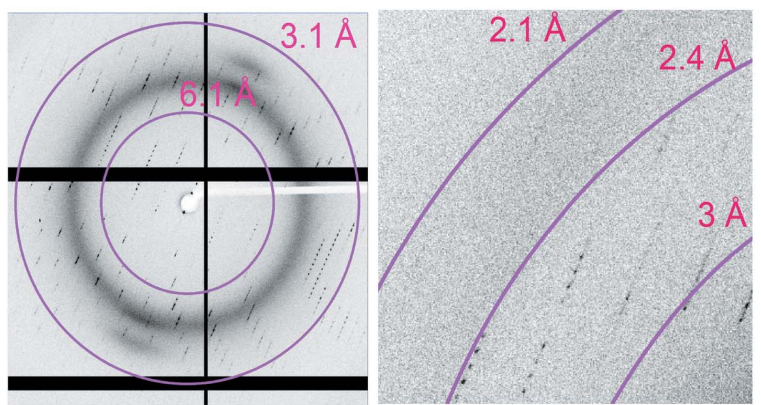

(b)

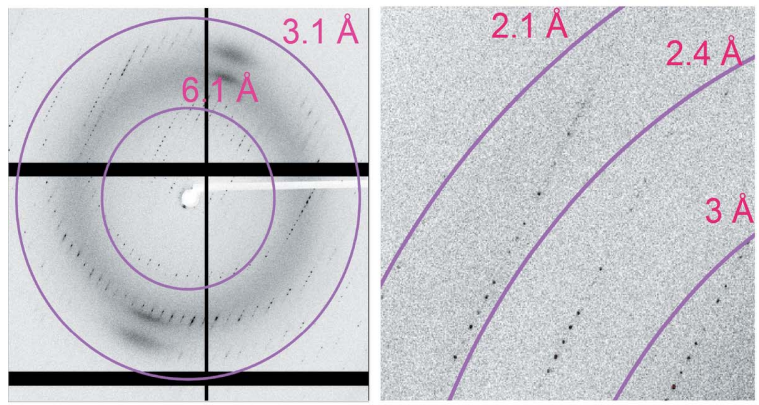

(c)
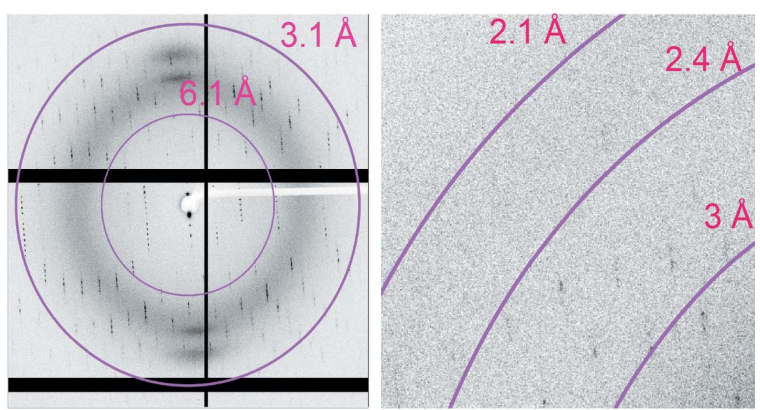

$(d)$

Figure 3

The diffraction images of the crystals with $(a)$ the host matrix, $(b)$ squalane, $(c)$ Paratone- $\mathrm{N}$ and $(d)$ liquid paraffin. The images were prepared by merging five consecutive diffraction images (corresponding to $1.0^{\circ}$ rotation). Views of the low-resolution region are shown in the left panels and magnified views of the high-resolution region are shown in the right panels. crystal centering was difficult. For liquid paraffin, the crystals were clearly observed, as when using squalane.

Full data sets were collected from these crystals. All crystals diffracted to high resolutions of $1.5-2.2 \AA$ (Fig. 3 and Supplementary Tables S1-S4). The mosaicity is related to the crystal quality and often increased during cryoprotectant treatment (Pflugrath, 2015). The average mosaicities for the crystals treated with oil were $0.13-0.15^{\circ}$. These values are comparable to that of $0.13 \pm 0.05^{\circ}$ for the untreated crystals [Fig. 4(a)]. The average Wilson $B$ factors, which are also related to the crystal quality, were $26-27 \AA^{2}$ for the crystals treated with oil and also comparable to that for the untreated crystals of $23 \pm 3 \AA^{2}$ [Fig. 4(b)]. From these facts, we concluded that the crystals retained their quality during the oil treatment.

\section{Discussion}

The method assessed herein was actually used in our recent studies for bR and heme A synthase (HAS) (Hasegawa et al., 2018; Niwa et al., 2018). In the case of bR, squalane was used for cleaning and cryoprotection (Hasegawa et al., 2018). The size of the crystals used by Hasegawa et al. was $300-400 \mu \mathrm{m}$, which was larger than the size of the crystals used in this work. However, the experiment was successful. In previous studies on bR, the host matrix has been removed from the crystal surface by means of enzymatic digestion (Nollert \& Landau, 1998) or detergent solubilization (Luecke et al., 1999). Compared with these previous approaches, our method can significantly reduce the treatment time from several hours or overnight to $3 \mathrm{~min}$ (Table 2). Moreover, our structures had the lowest average $B$ factor, indicating that the crystal quality was maintained during the oil treatment.

In the case of HAS, the structure of HAS was unknown and thus data sets for the experimental phasing were required. Moreover, the HAS crystals were small $(\sim 20 \mu \mathrm{m})$ and twinned. Therefore, we collected the full data set of HAS from one crystal using this oil cleaning method in order to facilitate

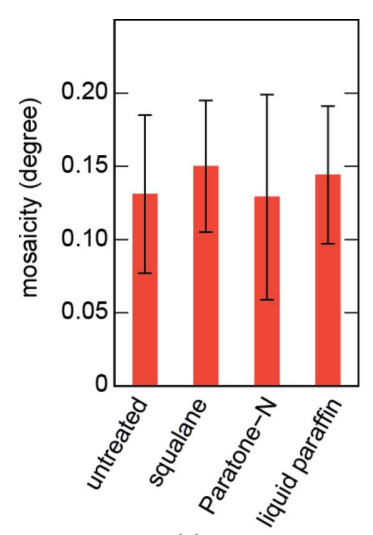

(a)

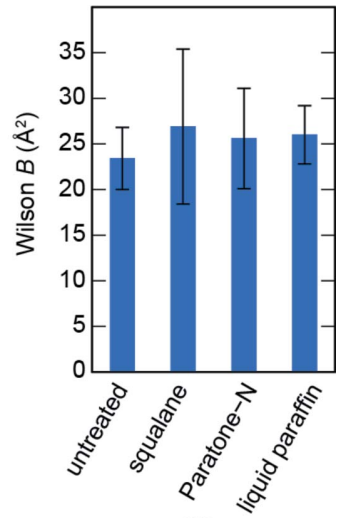

(b)
Figure 4

Effect on the crystal and data quality. (a) The mosaicities and (b) the Wilson $B$ factors for the crystals treated with each of the oils are shown. Error bars indicate standard deviations for the values shown in Supplementary Tables S1-S4. 


\section{cryocrystallography papers}

Table 2

The required time and the average $B$ factor of each method.

\begin{tabular}{llll}
\hline Method & Reference & Required time & Average $B$ factor $\left(\AA^{2}\right) \dagger$ \\
\hline Enzymatic digestion & Nollert \& Landau (1998) & Overnight & 35.0 (PDB ID 1qhj) \\
Detergent solution & Luecke et al. (1999) & Several hours-overnight & 30.0 (PDB ID 1c3w) \\
Oil cleaning & This work & $\sim 3$ min & 23.0 (PDB ID 5zil) \\
\hline
\end{tabular}

$\dagger$ The values are from the PDB entry in parentheses (Belrhali et al., 1999; Luecke et al., 1999; Hasegawa et al., 2018).

crystal centering and to decrease the background scattering. Squalane was also used for the harvest and cryoprotection because Paratone-N was not suitable for such small crystals. We found that the small crystals of HAS were stable in squalane for a short period of time. The crystal centering was possible since the oil on the nylon loops was completely transparent and the thickness of the oil film was appropriate. In the data collection from small crystals, the multicrystal data collection strategy is usually used to increase $I / \sigma(I)$ and the completeness of the data set (Zander et al., 2015). However, the merging of data from many crystals often affects the data quality indicators, such as $R_{\text {merge. }}$ In addition, when crystals are twinned or have low isomorphism, the merging method is difficult. In such situations, our method may be useful to collect full data sets from one crystal.

Most of the target proteins have no color, and the crystal centering problem is more severe for colorless crystals. The application to lysozyme crystals obtained by the LCP method (Aherne et al.,2012) as a model of colorless membrane protein crystals is shown in Supplementary Fig. S4. The oil-treated crystals were clearly visible, while untreated crystals were difficult to observe [Supplementary Fig. S4(a) and S4(b)]. Moreover, the scattering rings in the diffraction images were suppressed by oil treatment [Supplementary Figs. S4(c) and S4(d)]. Therefore, we conclude that this method is also useful for colorless crystals.

Squalane, Paratone-N and liquid paraffin did not affect crystals during harvesting, cleaning and flash-cooling. Paratone-N removed the host matrix most easily, while squalane and liquid paraffin were the easiest to use to mount and center crystals. On the basis of these results, it is advisable to test the oils and select the appropriate one for the experiment. This method will benefit studies of these crystals because correct centering contributes not only to the improvement of the signal-to-noise ratio but also to increasing the completeness of the data. Although membrane protein crystallography is challenging, we believe that this method will be of help in overcoming some of the difficulties.

\section{Acknowledgements}

We would like to thank Dr K. Miki and Messrs H. Jonotsuka and $\mathrm{N}$. Hasegawa for their contributions to the initial steps of this work. We are also grateful to Mr Imai for providing bR crystals. We thank the BL41XU beamline staff of SPring- 8 for their help with the X-ray data collections (proposal Nos.
2018B2706 and 2019A2560 to KT). Halobacterium salinarum was provided by the RIKEN BRC through the National BioResource Project of the MEXT, Japan.

\section{Funding information}

This work was supported by the Japan Society of Promotion of Science (JSPS) (KAKENHI grants JP15J02413 to SN, JP17H03643 to KT and JP25440020 to KT) and the grant program of the Research Foundation for Opto-Science and Technology (to KT).

\section{References}

Aherne, M., Lyons, J. A. \& Caffrey, M. (2012). J. Appl. Cryst. 45, 1330-1333.

Belrhali, H., Nollert, P., Royant, A., Menzel, C., Rosenbusch, J. P., Landau, E. M. \& Pebay-Peyroula, E. (1999). Struct. Fold. Des. 7, 909-917.

Caffrey, M. (2015). Acta Cryst. F71, 3-18.

Caffrey, M. \& Cherezov, V. (2009). Nat. Protoc. 4, 706-731.

Cherezov, V., Rosenbaum, D. M., Hanson, M. A., Rasmussen, S. G. F., Thian, F. S., Kobilka, T. S., Choi, H.-J., Kuhn, P., Weis, W. I., Kobilka, B. K. \& Stevens, R. C. (2007). Science, 318, 1258-1265.

Collaborative Computational Project, Number 4 (1994). Acta Cryst. D50, 760-763.

Dauter, Z. (2006). Acta Cryst. D62, 867-876.

Diederichs, K. (2010). Acta Cryst. D66, 733-740.

Hasegawa, N., Jonotsuka, H., Miki, K. \& Takeda, K. (2018). Sci. Rep. $\mathbf{8}, 13123$.

Hilgart, M. C., Sanishvili, R., Ogata, C. M., Becker, M., Venugopalan, N., Stepanov, S., Makarov, O., Smith, J. L. \& Fischetti, R. F. (2011). J. Synchrotron Rad. 18, 717-722.

Hirata, K., Yamashita, K., Ueno, G., Kawano, Y., Hasegawa, K., Kumasaka, T. \& Yamamoto, M. (2019). Acta Cryst. D75, 138-150. Kabsch, W. (2010). Acta Cryst. D66, 125-132.

Landau, E. M. \& Rosenbusch, J. P. (1996). Proc. Natl Acad. Sci. USA, 93, 14532-14535.

Luecke, H., Schobert, B., Richter, H.-T., Cartailler, J.-P. \& Lanyi, J. K. (1999). J. Mol. Biol. 291, 899-911.

Melnikov, I., Svensson, O., Bourenkov, G., Leonard, G. \& Popov, A. (2018). Acta Cryst. D74, 355-365.

Michel, H. (1983). Trends Biochem. Sci. 8, 56-59.

Niwa, S., Takeda, K., Kosugi, M., Tsutsumi, E., Mogi, T. \& Miki, K. (2018). Proc. Natl Acad. Sci. USA, 115, 11953-11957.

Nollert, P. \& Landau, E. M. (1998). Biochem. Soc. Trans. 26, 709-713.

Oesterhelt, D. \& Stoeckenius, W. (1971). Nat. New Biol. 233, 149-152.

Pebay-Peyroula, E., Rummel, G., Rosenbusch, J. P. \& Landau, E. M. (1997). Science, 277, 1676-1681.

Pflugrath, J. W. (2015). Acta Cryst. F71, 622-642.

Riboldi-Tunnicliffe, A. \& Hilgenfeld, R. (1999). J. Appl. Cryst. 32, 1003-1005.

Zander, U., Bourenkov, G., Popov, A. N., de Sanctis, D., Svensson, O., McCarthy, A. A., Round, E., Gordeliy, V., Mueller-Dieckmann, C. \& Leonard, G. A. (2015). Acta Cryst. D71, 2328-2343. 